\title{
Magneto-optic behaviour in the presence of surface plasmons
}

\author{
D M Newman ${ }^{1}$, M L Wears ${ }^{1}$, R J Matelon ${ }^{1}$ and I R Hooper ${ }^{2}$ \\ ${ }^{1}$ School of Engineering, Computing and Mathematics, University of Exeter, EX4 4QF, UK \\ ${ }^{2}$ School of Physics, University of Exeter, EX4 4QL, UK
}

Received 25 June 2008, in final form 22 July 2008

Published 7 August 2008

Online at stacks.iop.org/JPhysCM/20/345230

\begin{abstract}
Insight into the mechanism responsible for the enhanced level of magneto-optical (MO) activity observed from magnetic surfaces when supporting propagating surface plasmons (SPs) is provided by studies of ferromagnetic Ni and Co gratings where SPs are generated and interact magneto-optically at the air/ferromagnetic interface. These ferromagnetic gratings were subsequently coated with semi-transparent Ag layers. In these systems only some $30 \%$ of the light incident on the air/Ag interface is reflected; most is converted to surface plasmons whilst the remainder propagates to reflect off the ferromagnetic surface where it undergoes a MO interaction before returning through the Ag. The precise nature of the beam returned from the ferromagnetic and the MO signature imprinted on it depends on the relative magnitude of two driving sources: the $E$-field of the light wave and the $E$-field associated with SPs penetrating through the Ag to the ferromagnetic. Evidence is obtained indicating that the degree of MO enhancement correlates strongly with the magnitude of the intense electric field associated with the presence of SPs at the ferromagnetic surface, confirming previous experimental studies postulating that enhanced MO effects are not solely a consequence of the reduction in reflection that is experienced at surface plasmon resonance.
\end{abstract}

\section{Introduction}

Under certain specific conditions metallic surfaces can support the propagation of longitudinal surface waves in their freeelectron plasma. Collective electron oscillations of this nature when excited at a metal-dielectric interface are known as surface plasmons (SPs). On a structured metallic surface, in which the real part $\left(\varepsilon_{\mathrm{r}}\right)$ of the complex permittivity $\varepsilon(\omega)=$ $\varepsilon_{\mathrm{r}}+\mathrm{i} \varepsilon_{\mathrm{i}}$ takes negative values, SPs may be resonantly created at an angle $\theta$ from incident optical radiation by satisfying the relevant conservation laws. The angle at which SP's are created when the incident medium is air is given by equation (1).

$$
\sin \theta=\operatorname{Re}\left[\sqrt{\frac{\varepsilon(\omega)}{\varepsilon(\omega)+1}}\right] \pm \frac{m \lambda}{d}
$$

where $d$ is the grating period, $\lambda$ the incident wavelength and $m$ the integer determining the order of the process.

The transformation of photons to SPs in this manner not only results in a significant reduction in the intensity of the optical energy reflected from the surface, it also produces a very significant intensification of the electromagnetic field at the interface which decays exponentially into both bounding media [1]. This intensification is known to enhance certain surface effects including Raman scattering and second harmonic generation (SHG) [2-6]. Unlike the lowloss noble metals used predominantly to explore or exploit plasmonic behaviour, the complex permittivity of the three principle ferromagnetic elements possesses a large imaginary part $\left(\varepsilon_{\mathrm{i}}\right)$ so that any SPs excited on these materials are immediately subject to high non-radiative damping. Despite this apparently serious limitation previous studies on nickel gratings [5-9] and noble-metal/ferromagnetic layers in the Kretschmann-Raether configuration [10-15] have found deep SP resonances with reflection drops of up to $20 \%$ accompanied by greatly enhanced magneto-optical (MO) effects. In most of these studies however only indirect evidence supported the hypothesis that the enhancement in MO behaviour was primarily attributable to the SP electric field and not simply a consequence of the reduction in optical reflectivity. In this paper we extend the previous studies into SP behaviour on $\mathrm{Ni}$ to include Co and in attempting to resolve the ambiguity as to the precise origin of the MO enhancement also study, both experimentally and through modelling, the behaviour of a series of ferromagnetic gratings overcoated with thin layers of silver (Ag). 


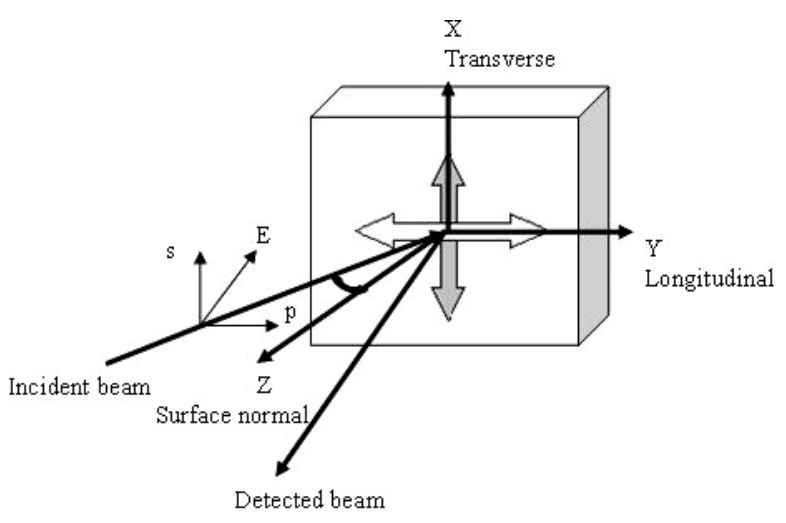

Figure 1. Shows the optical configuration for the MO geometries. The magnetic field is applied in the plane of the sample parallel to the plane of incidence, longitudinal effect or perpendicular to the plane of incidence, transverse effect.

\section{Experimental details}

The diffraction gratings used in this investigation were electrodeposited pure nickel with a pitch and depth of $1200 \mathrm{~nm}$ and $70 \mathrm{~nm}$ respectively. These gratings were subsequently DC sputter coated with thin metallic films of Co (>optical penetration depth) or 9, 16 and $30 \mathrm{~nm}$ of $\mathrm{Ag}$ using a Nordiko 2000 magnetron sputtering facility. The gratings were mounted on an automated measurement stage and orientated so that the grooves were orthogonal to the plane of incidence. Incident optical radiation over a wide spectral range (440-1064 nm) was provided by a sub miniature halogen lamp and a spot wavelength of $819 \mathrm{~nm}$ was afforded by a $40 \mathrm{~mW}$ Sony SLD202V-3 laser diode. Reflectance and MO measurements as a function of angle of incidence were subsequently conducted with p polarized radiation in the transverse Kerr configuration and longitudinal Kerr configuration figure 1.

\section{Results and discussion}

\subsection{Co coated gratings}

Comprehensive studies conducted on the $\mathrm{Ni}$ gratings and reported earlier [5,9] were extended by coating the $\mathrm{Ni}$ gratings with a thick Co film. Figure 2(a) shows the angular reflectivity spectrum for p-polarized radiation measured from these Co coated grating. Resonant creation of 1st order SPs given by equation (1) are clearly identified by the sharp drop in reflectivity which occur, for some given angle, for all wavelengths (for example SP1 for $1064 \mathrm{~nm}$ ). The SP excitation angle, is seen to decrease with increasing wavelength. Other, less dramatic, features in the reflectivity curves can be associated with 2 nd and 3rd order SP creation processes for example the less significant dips seen in the reflectivity curves for $1064 \mathrm{~nm}$ at $47^{\circ}$ (SP2) is due to the creation of 2nd order SPs where as the features in the $750 \mathrm{~nm}$ curve at $58^{\circ}$ and the $532 \mathrm{~nm}$ curve at $19^{\circ}$ (SP3) are associated with the creation of 3rd order SP. Simultaneous MO measurements of the fractional change in intensity $(\delta=\Delta R / R)$ on application of a magnetic field in the transverse Kerr configuration are shown in figure $2(\mathrm{~b})$.

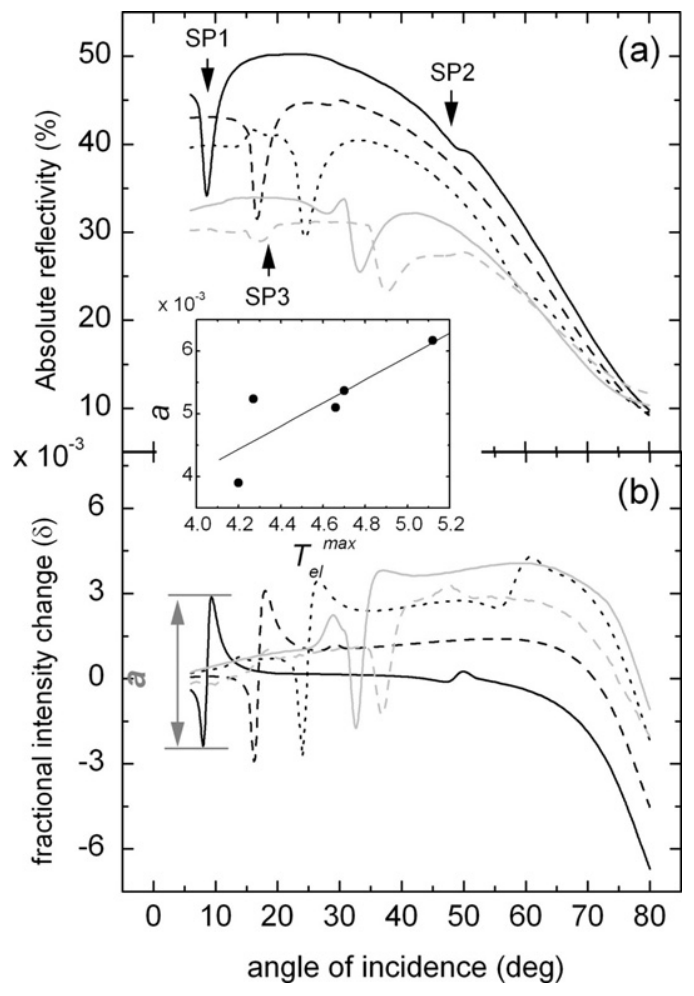

Figure 2. (a) Reflectivity spectrum and (b) fractional change as function of incidence angle and wavelength for Co grating for $1064 \mathrm{~nm}$ (black solid line), $900 \mathrm{~nm}$ (black dashed line), $750 \mathrm{~nm}$ (black dotted line), $600 \mathrm{~nm}$ (grey solid line), $532 \mathrm{~nm}$ (grey dashed line) and (inset) MO intensification, $a$, versus maximum field enhancement $\left(T_{\mathrm{el}}^{\max }\right)$.

A clear 'double spiked' resonance in $\delta$ is seen in the region of the 1 st order SP excitation angles where the fractional intensity change for a specular Co surface is featureless and tends to zero as normal incidence is approached [16].

Less significant resonances in $\delta$ can also be seen where 2nd and 3rd order SP are created as detailed above. Far away from the SP excitation angles the behaviour of grating and specular surfaces move into coincidence. In particular the magnitude of $\delta$ for both modulated and specular Co surfaces goes to a maximum at the pseudo-Brewster angle. This behaviour follows closely the multi-wavelength work in the transverse Kerr configuration reported on nickel gratings [5, 9] where the 'double spike' behaviour in $\delta$ was again clearly evident around the SP angles. As the coupling conditions denoted by the magnitude of the drop in reflectivity become increasingly favourable $\delta$ becomes up to ten fold larger compared to the value for a smooth Co surface endorsing the increases in $\delta$ reported in previous studies on Ni gratings [7, 9]. The foundation of this enhancement in MO behaviour seen originally in $\mathrm{Fe}$ [17] and now for both $\mathrm{Ni}$ and $\mathrm{Co}$ is, like the effects referred to in the introduction, presumed to lie in the intensification of the electromagnetic field associated with SP production. Although SPs on ferromagnetics are highly damped, their intensification of the electromagnetic field on the Ni gratings has already been well established through second harmonic and photo-acoustic studies [5, 6, 8]. Unfortunately, to unambiguously ascribe this as the sole or principle reason 
for the observation of enhanced values of $\delta(\Delta R / R)$ is not straightforward. Enhanced MO behaviour on thin films and gratings has also previously been explained $[18,19]$ as simply the consequence of the suppression of reflectivity by destructive interference and the coating of magnetic films with suitable dielectric layers is in fact a long established [20] technique for maximizing MO signals in optical recording.

In the comparative work on $\mathrm{Ni}$ gratings [5] in order to eliminate suppression in reflectivity as the sole mechanism for the observed enhancement in MO behaviour, attention was drawn to the reflectivity change upon application of a magnetic field $\Delta R$, proportional to the induced magnetic component of optical reflectivity. Measurements conducted in the transverse Kerr configuration using an ac technique in which the optical source is kept constant and the magnetic field modulated offer a direct account of $\Delta R$ [21]. The resonance in $\delta$ seen as a 'double spike' around the SP angle in figure 2 for Co gratings and in [5] for $\mathrm{Ni}$ gratings is again a feature of $\Delta R$ measurements on $\mathrm{Ni}$ gratings made in this direct fashion as a function of angle of incidence. The data in [5] also clearly show that the generated MO component is enhanced around the SP angle in comparison to a smooth surface.

However, whilst a reduction in, or suppression of, the normal Fresnel coefficient component as opposed to the induced magnetic component of optical reflectivity can yield enhanced magneto-optic behaviour it can not account for the 'double spike' feature and associated sign change evident in apparently all magneto-optic behaviour adjacent to the SP resonant angle. This requires that some degree of phase shift is also produced.

Optical phase shifts have been shown to occur when surface plasmons are generated [22-24]. Theoretical modelling and optical phase (no applied magnetic field) measurements performed on Ni gratings at $819 \mathrm{~nm}$ using an heterodyne optical phase measuring technique similar to that of Kabashin and Nikitin [25, 9] showed a phase change of $90^{\circ}$ as expected at the pseudo-Brewster angle but an optical phase change across the plasmon resonance of $15^{\circ}$ remote from the $180^{\circ}$ required to produce such behaviour as seen in $\delta$. Further measurements conducted on $\mathrm{Ni}$ gratings in the longitudinal Kerr configuration (figure 3) shows that the 'double spike' feature is recorded in the ellipticity, indicating a $180^{\circ}$ phase shift between the Kerr or magnetic component and the normal Fresnel component at the surface plasmon angle [26]. The transverse Kerr effect is seen as a change in the $r_{\mathrm{pp}}$ Fresnel coefficient, $r_{\mathrm{ss}}$ being unaffected by the application of a magnetic field, Penfold et al [27] measured the phase change associated with a specular Co film in the transverse Kerr configuration and showed that a change in phase of $90^{\circ}$ between the Fresnel and magnetic components in the transverse Kerr configuration produce a sign change in $\delta$ indicating that the 'double spike' behaviour seen around the SP angle is due to an $180^{\circ}$ phase shift between the Fresnel and magnetic components of the reflected radiation.

As the SPs couple to the optical field at the Co/air interface the intensity of the electric field at the metal dielectric interface increases, energy conservation [28] can be used to estimate

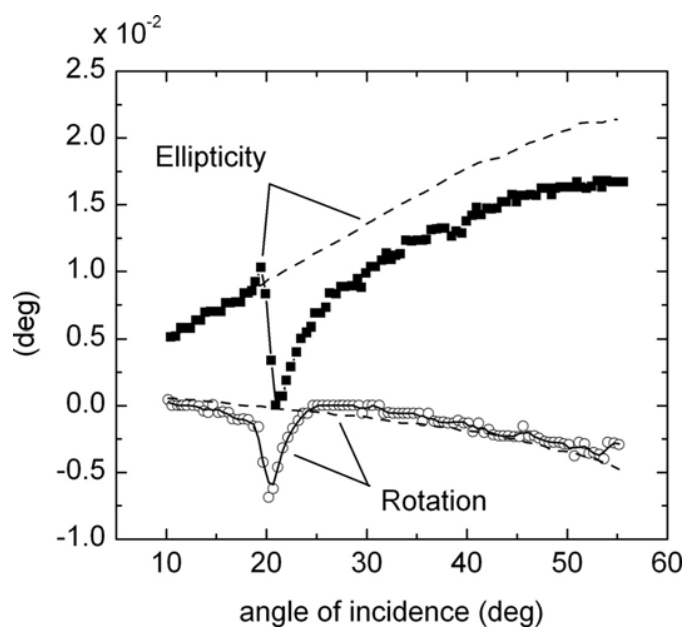

Figure 3. Longitudinal rotation and ellipticity measured at $819 \mathrm{~nm}$ from the Ni grating (no overcoat) as a function of angle of incidence; data recorded from a flat $\mathrm{Ni}$ surface is also shown (dash line) for comparison.

this enhancement $\left(T_{\mathrm{el}}\right)$. Inserting permittivity values from Newman [29] and Johnson [30] into equation (2)

$$
T_{\mathrm{el}}=\frac{2\left|\varepsilon_{\mathrm{r}}\right|^{2} \cos \theta(1-R)}{\varepsilon_{\mathrm{i}}\left(\left|\varepsilon_{\mathrm{r}}\right|-1\right)^{1 / 2}}
$$

where $R$ is the measured total power reflectance and all other terms are as previously described, yields the field enhancement at the air/Co surface for all the wavelengths displayed in figure 2. The maximum field enhancement $\left(T_{\mathrm{el}}^{\max }\right)$ occurs at the 1st order SP excitation angle, the increase in $\delta$ around this angle is seen by comparison of figures 2(a) and (b) and displayed in figure 2 (inset) to be dependant on $T_{\mathrm{el}}^{\max }$ endorsing the earlier hypothesis that the enhancement in the electric field is associated with the increase seen in $\delta$ at the SP excitation angle.

\subsection{Ag coated gratings}

To probe further the nature of the relationship between the enhancement of MO effects and SP field intensification, Ni gratings were now coated with various thicknesses of silver. In samples such as this, the interfaces that permit a magnetooptic interaction and those that support SP behaviour are physically separated by the thickness of the Ag film. Magnetooptic behaviour will only be observed when the Ag film is thin enough for optical radiation to penetrate to and return from the ferromagnetic surface but the generation of SPs on this surface by the optical beam incident through the Ag is precluded because the conditions referred to previously are not met at this interface. Depending on the precise thickness of the Ag film the electrons re-radiating optical radiation from the ferromagnetic surface are however still subject to two driving terms, the $E$-field of the light wave incident through the $\mathrm{Ag}$ and the $E$-field associated with the SPs at the air/Ag interface penetrating through the $\mathrm{Ag}$ and decaying to the ferromagnetic surface for clarification see figure 4 . 


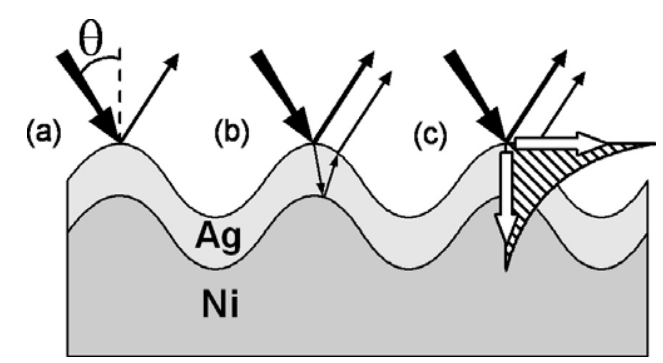

Figure 4. Schematic showing how incident radiation is transmitted through Ag overlayer to interact with $\mathrm{Ni}$ surface. When radiation is incident at the plasmon angle $\theta$ it interacts with the grating in a number ways (a) radiation is partly reflected from the Ag surface, (b) radiation decays through the $\mathrm{Ag}$ to the Ni surface and is reflected back through the $\mathrm{Ag}$, (c) radiation couples to the SPs which then travel along the surface, the electromagnetic field associated with the SPs then decays through the Ag surface to the Ni surface.

The precise nature of the beam returned from the ferromagnetic and the magneto-optic signature imprinted on it therefore depends on the relative magnitude of these two driving sources which of course vary with the thickness of the Ag layer.

After using an AFM to precisely determine the physical profiles of the coated structures, calculated reflectivity curves were generated using the grating profile in figure 5(a) and a modelling code based on a combination of the differential formalism of Chandezon et al [31] and a scattering matrix approach [32]. The method has since been improved and applied to study a wide range of diffractive grating systems [33-41]. The essence of the method is to map the periodically structured surface onto a flat plane by the use of a nonorthogonal curvilinear coordinate transformation. Maxwell's equations are then solved in the new coordinate system. Initial extensions to describe multilayer systems were based on the use of transfer matrices in order to associate the eigenmodes across the interfaces [42]. However, this method can become numerically unstable when the layer thickness become large, and therefore the use of scattering matrices was introduced by Preist et al [31] in order to overcome this limitation. Matching the tangential components of the fields across the interface, the scattering matrix of the interface is calculated, and is defined by

$$
\left(\begin{array}{c}
C_{T}^{+} \\
C_{S}^{-}
\end{array}\right)=S\left(\begin{array}{c}
C_{T}^{-} \\
C_{S}^{+}
\end{array}\right)
$$

where $C_{T}^{+}\left(C_{T}^{-}\right)$is a vector containing the coefficients of upward (downward) going eigenmodes in the top medium and $C_{S}^{+}\left(C_{S}^{-}\right)$is a vector containing the coefficients of upwards (downwards) going eigenmodes in the substrate. The reflection and transmission coefficients of the grating system can then be calculated using this scattering matrix, along with any required field profiles. Figure 5 shows the close comparison obtained between the output of this model and experimentally measured values for the bare and $\mathrm{Ag}$ coated $\mathrm{Ni}$ grating at $819 \mathrm{~nm}$. The absence of sharp features in the experimental reflectivity curves at angles near $19^{\circ}$, but revealed by theoretical modelling, is attributed to a possible slight mismatch between calculated
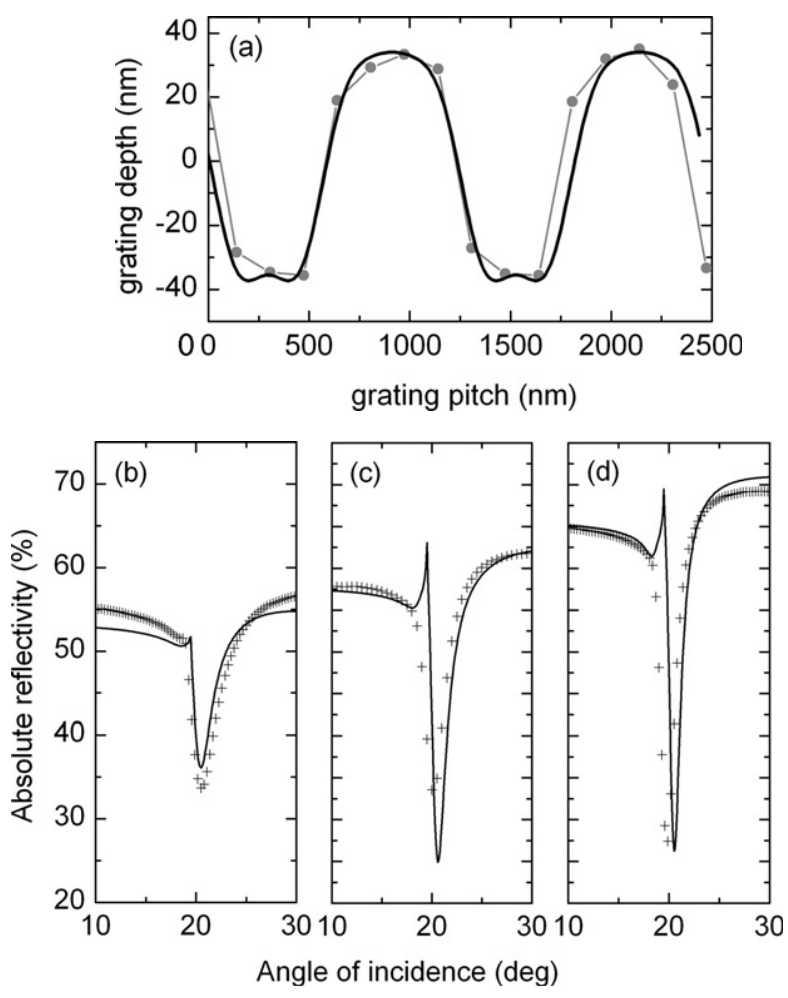

Figure 5. (a) Grating profile measured on AFM (•) and used in simulations (-). Reflectivity as a function of incident angle measured $(++++)$ and calculated $(-)$ reflectivity curves at $819 \mathrm{~nm}$ for Ni gratings with (b) no overcoat, (c) $9 \mathrm{~nm}$ and (d) $16 \mathrm{~nm}$ of silver.

and AFM grating profiles, fitted as a Fourier series of four harmonics, and probable angle spread of the incident beam. One of these features, at $18.3^{\circ}$ in figures $5(\mathrm{c})$ and (d), is attributed to second order SP generation processes seen as a drop in reflectivity, as with first order processes, whereas the sharp increase in reflectivity at $19.5^{\circ}$ is associated with diffraction edges commonly seen as diffraction orders, +1 order in this case, are passing off over the grating horizon and often referred to as Rayleigh anomalies. Experimentally these features overlap with the reflectivity drop associated with first order SPs leading to a widening of the reflectivity curve in the range of incidence $\left[17^{\circ}, 22^{\circ}\right]$ compared to theoretical data.

The depth of the reflectivity curves in figure 5 provide an indication as to the magnitude of the enhanced electromagnetic field due to the creation of SPs, equation (2) can again be used together with permittivity values taken from Palik [43] to estimate the maximum field enhancement at the plasmon angle $\left(T_{\mathrm{el}}^{\max }\right)$. At the air/Ni surface of the bare $\mathrm{Ni}$ grating a value of 2.5 was calculated for $T_{\mathrm{el}}^{\max }$ in agreement with previous analysis on the Ni grating using a photo-acoustic technique [8] whilst $T_{\mathrm{el}}^{\max }$ at the $9 \mathrm{~nm}$ and $16 \mathrm{~nm}$ air/Ag surfaces were an order of magnitude larger 22.53 and 22.05 respectively. If the enhancement in the MO effect is purely a consequence of the field enhancement at the metal dielectric interface then expected values for $\delta$ on the Ag coated gratings would be greater than for the bare Ni gratings, whereas experimental values displayed for $\delta$ in figure 6 are of the same order of magnitude. 


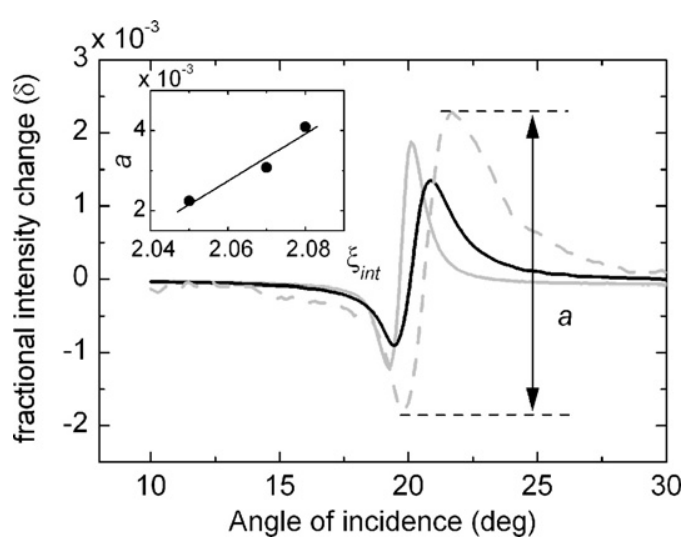

Figure 6. Fractional change $\delta$ for Ni gratings with (grey dashed line) no overcoat, (black solid line) $9 \mathrm{~nm}$ and (grey solid line) $16 \mathrm{~nm}$ of silver, (inset) MO intensification, $a$, versus average field intensification, $\xi_{\text {int }}$.

Films coated with $30 \mathrm{~nm}$ of silver (>than the optical penetration depth) showed no MO response. Again as mirrored in the MO curves from the $\mathrm{Co}$ and $\mathrm{Ni}$ gratings displayed in figures 2 and 3 the 'double spike' behaviour is evident in the MO results from the Ag coated gratings displayed in figure 6 indicating a phase change in the reflected light.

Finally, to try and explain the ambiguity between the magnitude of $\delta$ and the field intensification due to SP creation, the time averaged electric field distribution over the grating was calculated using the modelling code described above, with light incident at the 1 st plasmon resonance angle, and a value of one corresponding to the field amplitude of the incident field. The associated field maps (figure 7) revealed as expected larger fields at the air/Ag grating surface when compared to the bare nickel grating surface. However the field intensity at the Ag surface is seen to decay through the Ag layer to the $\mathrm{Ni}$ interface. Integration of the electric field distribution sensed by the Ni interacting with penetrating optical radiation $\left(\xi_{\text {int }}\right)$, in figure 7 i.e. within the optical skin depth of the gratings, over a full grating period reveals that the average intensification in the bare Ni surface $\left(\xi_{\text {int }}\right)$ due to the SP is approximately the same as that seen in the Ni surface of the Ag coated gratings after the SP field has decayed through the Ag to the Ni. Careful examination shows that the magnitude of the MO enhancement $(a)$ is linear with $\left(\xi_{\text {int }}\right)$ as seen in figure 6 (inset) confirming the results from the Co gratings displayed in the inset of figure 2 that $\delta$ is proportional to the enhanced field generated by SPs.

\section{Conclusions}

Surface plasmons, observed as a drop in reflectivity over a small angular range, were generated on ferromagnetic and silver coated ferromagnetic gratings. The resulting intensified electric field at the ferromagnetic surface and its significance in enhancing MO effects was deduced theoretically and modelled to experimental data to offer proof that the electric field intensification associated with the generation of surface plasmons on structured surfaces is responsible for the recorded enhanced MO behaviour. The magnitude of the intensification
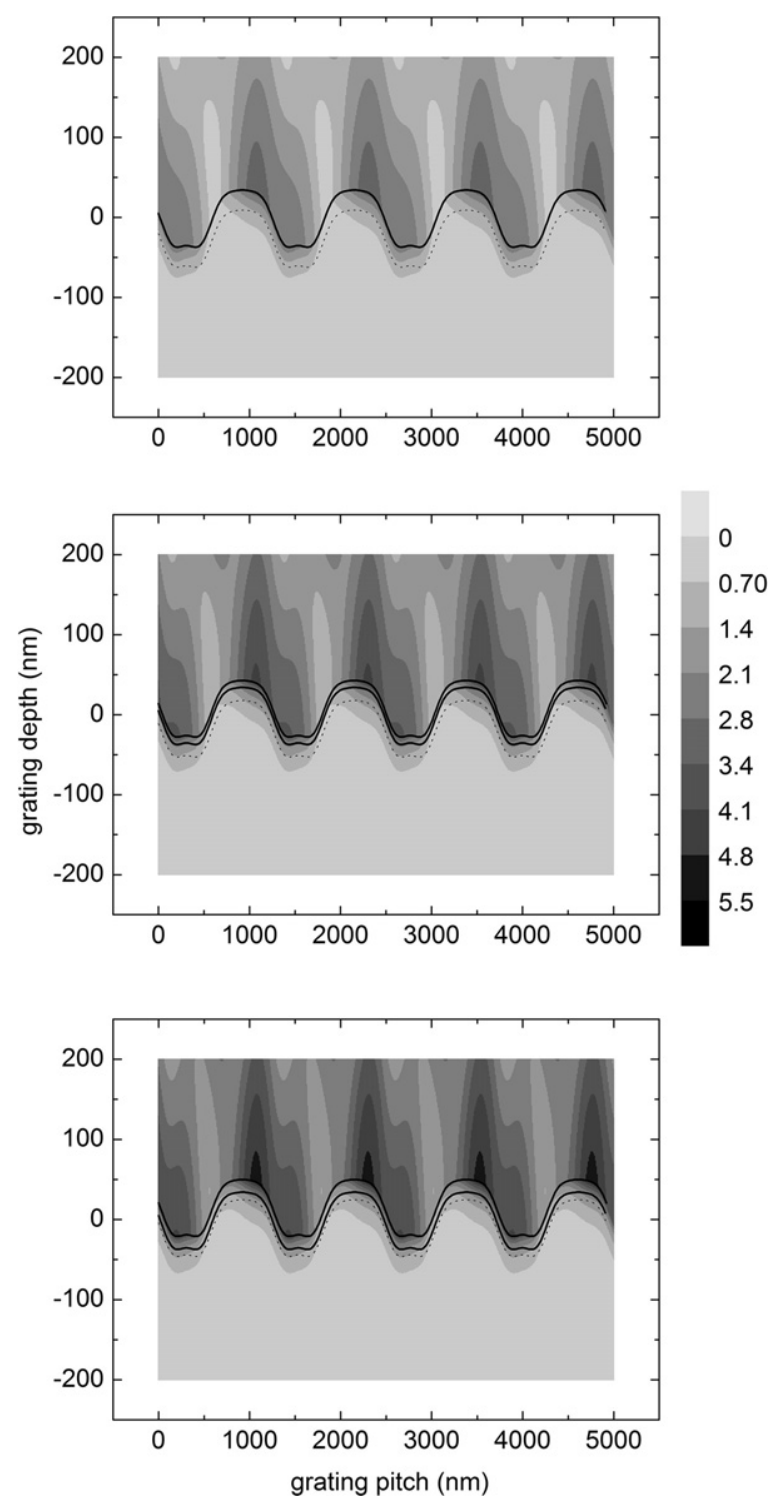

Figure 7. The time averaged electric field distribution over the $\mathrm{Ni}$ gratings with (a) no overcoat, (b) $9 \mathrm{~nm}$ and (c) $16 \mathrm{~nm}$ of silver, when light is incident at the 1 st plasmon resonance angle.

of MO effects is shown to be highly sensitive to the electric field sensed by the magnetic layer interacting with the incident optical radiation.

\section{References}

[1] Raether H 1988 Surface Plasmons on Smooth and Rough Surfaces and on Gratings (London: Springer)

[2] Primeau N, Coutaz J L and Abello L 1983 J. Appl. Phys. 735158

[3] Numata H 1982 Phys. Soc. Japan 512575

[4] Coutaz J L, Neviere M, Pic E and Reinisch R 1985 Phys. Rev. B 322227

[5] Newman D M, Wears M L, Matelon R J and McHugh D 2002 Appl. Phys. B $\mathbf{7 4} 719$

[6] Newman D M, Wears M L, Atkinson R and McHugh D 2002 J. Magn. Magn. Mater. 242-245 980

[7] Carey R, Newman D M and Matelon R J 2001 J. Magn. Soc. Japan 25271 
[8] Matelon R J, Newman D M and Wears M L 2004 Rev. Sci. Instrum. $\mathbf{7 5} 260$

[9] Matelon R J 2003 The interaction of light with periodic magnetic structures $P h D$ Thesis Coventry University

[10] Safarov V I, Kosobukin V A, Hermann C, Lampel G, Peretti J and Marliere C 1994 Phys. Rev. Lett. 733584

[11] Kosobkin V A 1996 J. Magn. Magn. Mater. 153397

[12] Richard N, Dereux A, David T, Bourillot E, Goudonnet J P, Scheurer F, Beaurepaire E and Garreau G 1999 Phys. Rev. B $\mathbf{5 9} 5936$

[13] Hermann C, Kosobukin V A, Ampel G, Peretti J, Safarov V I and Bertrand P 2001 Phys. Rev. B 64235422

[14] Bonod N, Reinisch R, Popov E and Neviere M 2004 J. Opt. Soc. Am. B 21791

[15] Matelon R J, Newman D M and Wears M L 2002 Trans. Magn. Soc. Japan 2216

[16] Sokolov A V 1997 Optical Properties of Metals (London: Blackie)

[17] Carey R, Loken M, Newman D M and Thomas B W J 1997 J. Phys. D: Appl. Phys. 30166

[18] Eremenko V, Novosad V, Pishko V, Geoffroy O, Souche Y and Pannetier 1997 JETP Lett. 66494

[19] Souche Y, Tufaile A P B, Santi C E, Novosad V and Santos A D 1998 J. Magn. Magn. Mater. 177-181 1277

[20] Hunt R P 1967 J. Appl. Phys. 381215

[21] Neal K F J 1963 The magneto-optical behaviour of ferromagnetic metal PhD Thesis University of London

[22] Shen S, Liu T and Guo J 1998 Appl. Opt. 371747

[23] Guo J, Zhu Z, Deng W and Shen S 1998 Opt. Eng. 372998

[24] Kochergin V E, Beloglazov A A, Valeiko M V and Nikitin P I 1998 Quantum Electron. 28444
[25] Kabashin A V and Nikitin P I 1997 Quantum Electron. 27653

[26] Atkinson R, Salter I W and Xu J 1992 Appl. Opt. 314847

[27] Penfold C, Collins R T, Tufaile A P B and Souche Y 2002 J. Magn. Magn. Mater. 242-245 964

[28] Weber W H and Ford G W 1981 Opt. Lett. 6122

[29] Newman D M 1978 Magneto-optical properties of thin Co films PhD Thesis Lanchester Polytechnic

[30] Johnson P B and Christy R W 1974 Phys. Rev. B 95056

[31] Chandezon J, Dupuis M T, Cornnet G and Maystre D 1982 J. Opt. Soc. Am. 72839

[32] Preist T W, Cotter N P K and Sambles J R 1995 J. Opt. Soc. Am. A 121740

[33] Li L 1994 J. Opt. Soc. Am. A 112816

[34] Harris J B, Preist T W, Sambles J R, Thorpe R N and Watts R A 1996 J. Opt. Soc. Am. A 132041

[35] Preist T W, Cotter N P and Sambles J R 1995 J. Opt. Soc. Am. A 121740

[36] Granet G 1995 Pure Appl. Opt. 4777

[37] Wanstall N P, Preist T W, Tan W C, Sobnack M B and Sambles J R 1998 J. Opt. Soc. Am. A 152869

[38] Hooper I R and Sambles J R 2002 Phys. Rev. B 65165432

[39] Hooper I R and Sambles J R 2002 Phys. Rev. B 66205408

[40] Hooper I R and Sambles J R 2003 Phys. Rev. B 67235404

[41] Hooper I R and Sambles J R 2004 Phys. Rev. B 70045421

[42] Chandezon J, Dupuis M T, Cornet G and Maystre D J 1982 Opt. Soc. Am. 72839

[43] Palik E D 1985 Handbook of Optical Constants of Solids (London: Academic) 